\title{
5 Choice of Solvent in Liquid-Phase Microextraction
}

\author{
Shayessteh Dadfarnia* and Ali Mohammad Haji-Shabani \\ Department of Chemistry, Faculty of Science, Yazd University, Yazd, 89195-741 Iran \\ ^e-mail address: sdadfarnia@yazduni.ac.ir
}

\subsection{Introduction}

Liquid-phase microextraction (LPME) is a miniaturized sample preparation technique that emerged in the mid-to-late 1990s (Liu \& Dasgupta, 1996; Jeannot \& Cantwell, 1996). In LPME, a microliter amount of water-immiscible solvent is used to extract analytes from aqueous samples. The principle of LPME is similar to the traditional liquid-liquid extraction. Thus, the choice of appropriate extracting solvent is an important aspect of a successful LPME. There are several peripheral properties of solvents which are of interest in selecting a solvent (Barwick, 1997) but do not directly affect the separation. These factors are frequently conflicting, and certainly no single substance would ordinary possess every desired characteristic. The final choice should be made after comparing the different physical properties of the solvents available and the required criteria for different modes of LPME to achieve good selectivity, sensitivity and precision. In this chapter, the relative importance of the various factors in choosing a solvent for LPME are considered and briefly discussed.

\subsection{Relevance of Physicochemical Properties in Extractant Phase Selection}

\subsubsection{Solubility}

This is the first property that must be considered in deciding the applicability of a solvent, and it refers to the ability of a solvent to dissolve considerable amounts of the analyte. The solute should be very soluble in the extracting phase. Generally, the reason for dissolution is thermodynamic, i.e., when the process is energetically favorable it will occur. However, kinetics can also play a role and solutes that are poorly soluble at room temperature may be dissolved at high temperature. In the qualitative selection of the appropriate solvent, one can use the rule "like dissolves like"; however, there are many parameters that have been used to describe the attractive forces (dispersion, dipole and hydrogen bonding) present within a solvent or liquid. For example, the refractive index value is a good measure of the tendency of compounds to interact by dispersion forces (Barwick 1997), i.e., the greater the refractive index values of the compounds, the stronger the dispersion interaction. Polarity is 
often used to predict the solubility of a compound, but unfortunately the concept is not straightforward. Among different classifications of solvent polarity, the Hildebrand polarity scale or Hildebrand solubility parameter $d$ is reported as being the most widely applied index of solvent and solute polarity. Table $\mathbf{5 . 1}$ lists d values for a number of solvents along with some of their physical properties such as boiling point, dielectric constant, surface tension, density and vapor pressure. Values of $d$ can be estimated for other compounds by bearing in mind that homologues of polar compounds tend to have similar but slightly lower values of $d$ as molecular weight increases. The solubility of a solute in a solvent is maximized when they have the same $d$ values. In general, two liquids are miscible if the difference in $d$ value is less than 3.4 units. Two solvents whose $d$ values are higher or lower than that of a given solute can be blended to give a mixture with a d value equal to that of solute, thus maximizing the solute solubility. However, there are exceptions to the Hildebrand solubility parameter especially with polar solvents and solutes; therefore, it is often worth testing solubility or solvent miscibility on a small scale even if data are available.

\subsubsection{Distribution Coefficient}

A distribution coefficient is defined as the ratio of the total concentration of solute in the organic phase to the total concentration of solute in the aqueous phase at equilibrium. It gives a measure of the difference in the solubility of the solute in these two phases. A distribution coefficient value that is greater than unity implies that the solute has a higher affinity for the organic phase than the aqueous phase. The higher the distribution coefficient, the more easily the solute will be extracted into the extracting phase. So, for the extraction of a single solute from the aqueous phase, a large distribution coefficient is desirable to achieve a high enrichment factor (EF) (defined as the ratio of the analyte concentration in the extraction phase to its initial concentration in the sample solution) and extraction efficiency. Thus, the distribution parameter, which depends to a large extent on the type and nature of the extracting phase, is a key variable and it is necessary to emphasize the different extracting phases that can be used.

\subsubsection{Selectivity}

If the aqueous phase contains more than one solute, then in deciding on the applicability of a solvent the selectivity must be considered. The selectivity refers to the ability of a solvent to extract one solute (x) in preference to another (y) and is defined as the value of the distribution coefficient of $\mathrm{x}$ to the distribution coefficient of $\mathrm{y}$. The most suitable solvent from this point of view would dissolve a maximum of one 


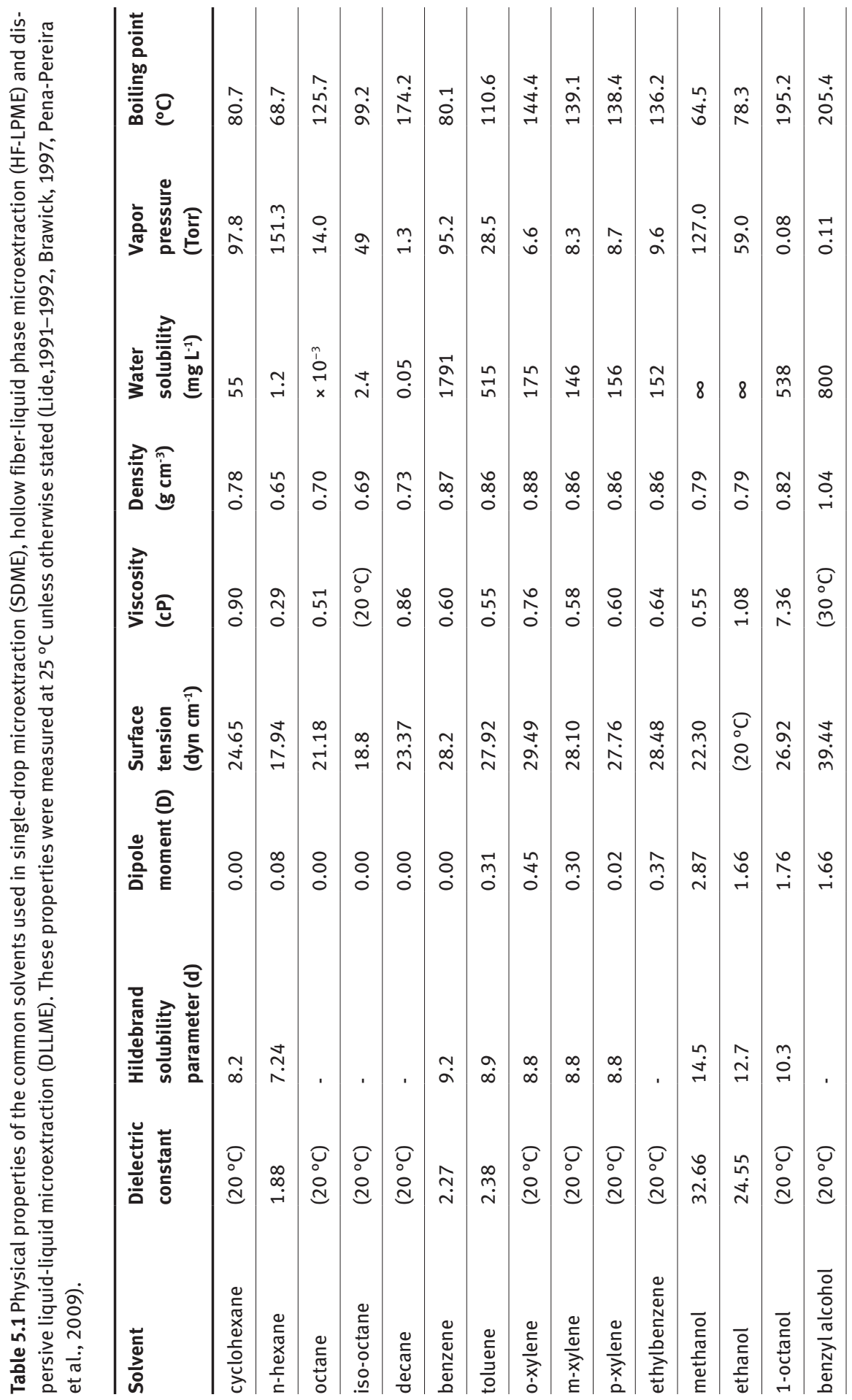




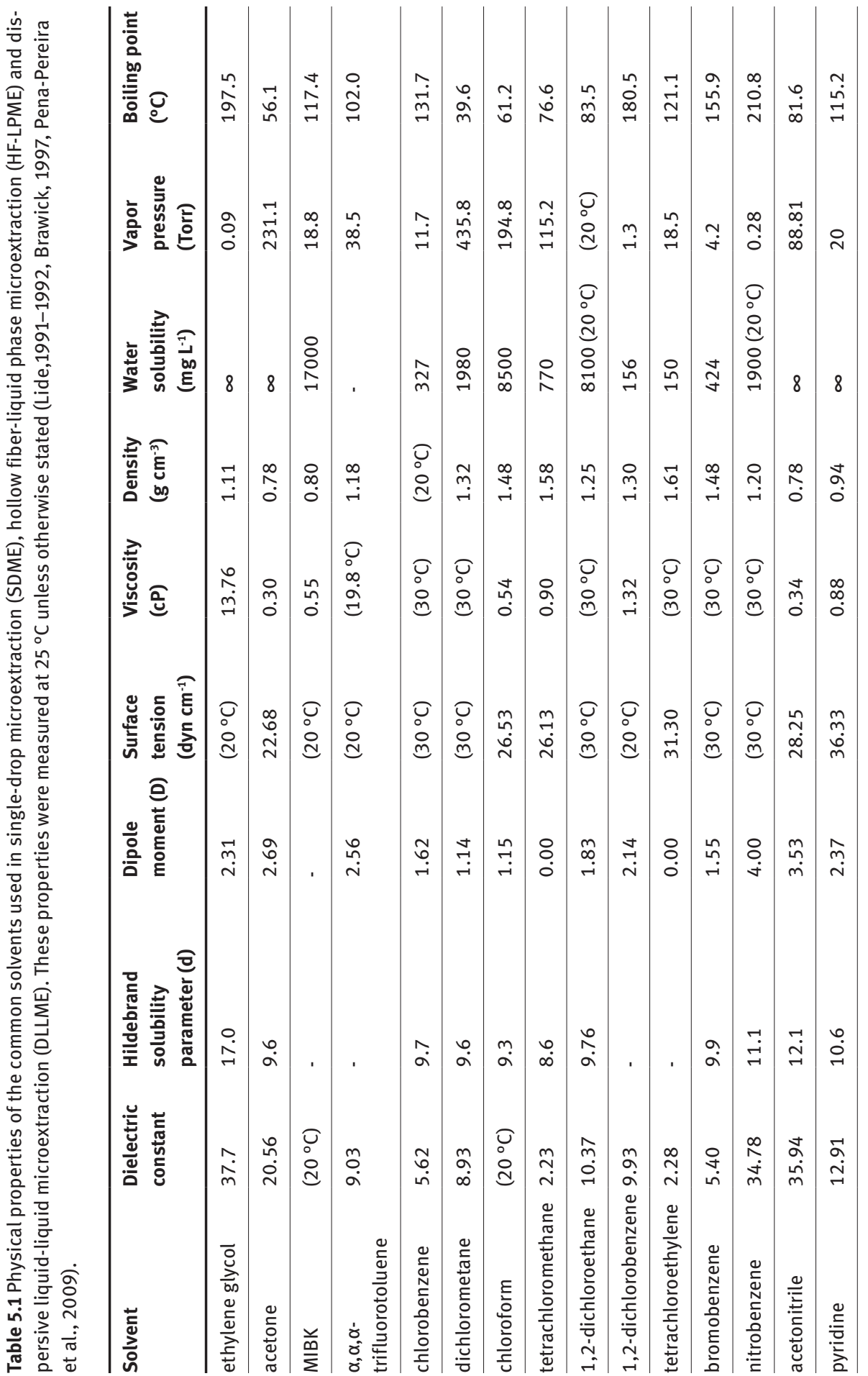




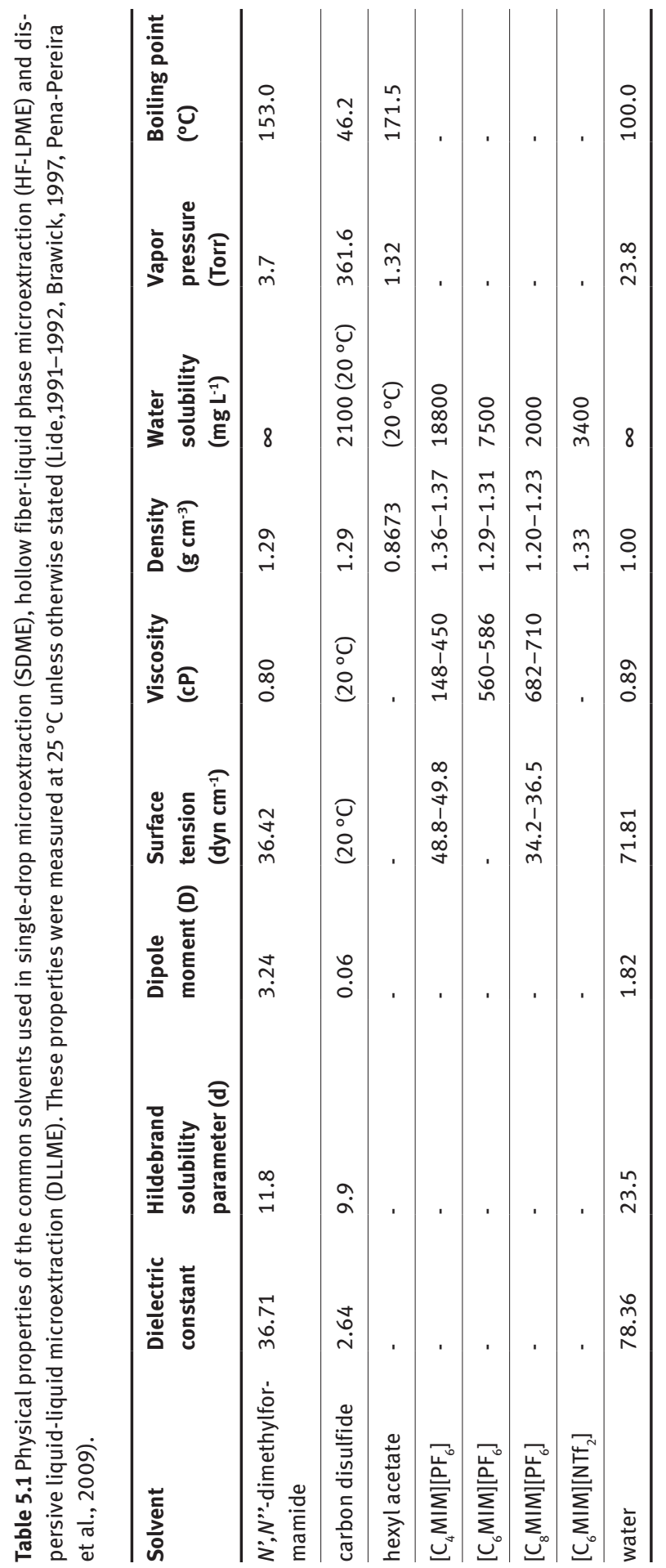


solute and a minimum of the other. In this regard, the closeness of the polarity of the solvent to the analyte is important. If the polarity of the analyte of interest differs significantly from that of other components, a solvent with polarity close to the analyte will significantly extract the analyte without extraction of other components. In some cases, variation in dispersion interactions may affect the selectivity. Normally dispersion interactions play a minor role in solvent selectivity, but it is worth considering in the separation of solutes with different refractive indices. Thus, replacing a solvent with another solvent of similar polarity but different refractive index may enhance the solvent selectivity. For a useful extraction operation, the selectivity must be greater than unity. A selectivity of unity means that the solvent have no preference for the solutes and no separation is possible.

\subsubsection{Immiscibility}

For a clean separation, the extraction solvent should have low solubility in the aqueous phase. If the solubility of the organic phase in the aqueous phase is significant, complete recovery of analyte is not possible and an additional separation step is required. One way of arriving at an idea about the degree of immiscibility is through a comparison of the dipole moment of the liquid with that of water $(\mu=1.84$ Debye). Solvents such as benzene, hexane and carbon tetrachloride with dipole moments of zero have low water solubility (less than $0.1 \mathrm{~g} / 100 \mathrm{~g})$, whereas solvents such as diethyl ether $(\mu=1.2$ Debye), ethyl acetate ( $\mu=1.8$ Debye), or $n$-butyl alcohol $(\mu=1.7$ Debye) have relatively high aqueous solubil-

ity $(6.90,7.94$, and $7.80 \mathrm{~g} / 100 \mathrm{~g}$, respectively). This generalization has its exceptions, of course; chlorobenzene with a dipole moment of 1.6 Debye has an aqueous solubility less than that of benzene.

\subsubsection{Density}

Solvent density is critical in conventional liquid-liquid extraction, as well as in many LPME approaches. However, the relevance of the extractant phase density is lower in SDME and HF-LPME techniques. A large difference in densities between the extracting and aqueous phase permits a clean-cut phase boundary between the two phases. It should be noted that it is insufficient to examine only the relative densities of the solution to be extracted and the pure extracting solvent, since on mixing the mutual solubility of the two will alter the individual densities. 


\subsubsection{Interfacial Tension}

Solvent interfacial tension affects the dispersion of one liquid to another and the ease of the separation of two phases. For rapid coalescence of emulsion and phase separation a high interfacial tension between immiscible phases is required. However, too high an interfacial tension may lead to difficulties in the adequate dispersion of two phases and consequently mass transfer efficiency. Thus, a compromise may be necessary in the interfacial tension; it must be high enough to prevent the formation of stable emulsion and low enough to permit adequate dispersion of one liquid in the other to occur. As the information regarding the liquid interfacial tension for complete ternary systems is limited, one should use the differences in the surface tension with air of the contacted liquid as a rough guide to estimate the order of magnitude of interfacial tension.

\subsubsection{Chemical Reactivity}

The reaction between the solvent and other component of the system is undesirable. Thus, the solvent must be chemically stable and inert toward the sample matrices and materials used in the construction of extraction devices and should not polymerize, condense or decompose under the extraction conditions. However, on occasion, the formation of a chemical compound between the analyte and solvent as part of the extraction process is desirable and the rate and even the extent of extraction may be enhanced.

\subsubsection{Corrosiveness}

In order to reduce the cost of the extraction equipment, the solvent should be inert and cause no severe corrosion difficulties with common materials of construction. Thus, expensive alloys and other unusual materials should not be required.

\subsubsection{Viscosity, Boiling Point and Vapor Pressure}

Solvent viscosity also affects the extraction kinetics in LPME. The viscosity of the solvent should be low to permit good contact between the two phases while mixing and to allow rapid settling out of the two liquids after shaking. Thus, a high viscosity leads to difficulties in dispersion and the mass transfer rate.

The solvent should have a sufficiently high boiling point and low vapor pressure so that evaporation of the solvent is not a problem. A solvent with high vapor pressure may cause difficulties in the storage and operation of extraction at atmospheric pressure. 


\subsubsection{Availability and Cost}

The solvent should be readily available in a sufficient state of purity for convenient use. A rare solvent or one that requires long and elaborate purification process before it can be used is not going to become popular and widely used, regardless of its extraction power.

\subsubsection{Other Criteria}

The freezing point, flammability and toxicity of the solvent must be low. The freezing point of the solvent must be low enough so that it can be conveniently stored. The low flammability and toxicity of the solvent is desirable for occupational health and safety consideration. However, in LPME, in contrast to liquid-liquid extraction, the recoverability of the solvent is not important as the use of solvent is reduced to microliter levels.

\subsection{Extracting Solvents for Liquid-phase Microextraction}

The extracting solvents used in LPME can be divided into two general groups, namely organic solvents and ionic liquids (ILs).

To date, the most common extracting phases with LPME methodology are organic solvents. The proper choice of the organic solvent should be based on the required criteria for different modes of LPME methods and physical properties of the organic solvents, the aspects of which will be considered later.

ILs are generally composed of bulky, nonsymmetrical organic cations, such as imidazolium, pyrrolidinium, pyridinium, ammonium or phosphonium and many different inorganic or organic anions such as tetrafluoroborate and bromate anions. ILs are gaining widespread recognition as green solvents in chemistry. The unique properties of ILs, such as negligible vapor pressure, good thermal stability, tunable viscosity and miscibility with water and organic solvents and their good extractability for a wide range of organic compounds and metal ions depends mainly on the nature and size of their cationic and anionic constituents. Some ILs are suitable for different modes of LPME due to their immiscibility with water, which permits the formation of two-phase systems, and the high affinity of their organic constituents (Han et al., 2012). However, it should be noted that although room temperature ILs based on alkyl imidazolium hexafluorophosphate have been used by many researchers as green solvents, these compounds may be potentially toxic due to the instability of the $\left[\mathrm{PF}_{6}\right]$ anion toward hydrolysis in contact with moisture, which results in forming some volatile species, including $\mathrm{HF}$ and $\mathrm{POF}_{3}$ (Swatloski et al., 2003). In the following section the main required criteria for sol- 
vents in different modes of LPME as well as the most common solvents used will be considered.

\subsubsection{Extractant Phases for Single-drop Microextraction}

The most important parameters that must be considered in the selection of a solvent for direct single-drop microextraction (direct-SDME) and continuous flow microextraction, where the drop is immersed into the aqueous solution, are selectivity, incidence of drop loss, rate of drop dissolution, extraction efficiency and solvent toxicity. Thus, a solvent with relatively high boiling point and high surface tension is required. The high boiling point solvent reduces the possibility of evaporation losses and prevents the possibility of bubble formation inside of the drop whereas a high surface tension increases the cohesive forces at the interface which reduce solvent solubilization (Psillakis \& Kalogerakis, 2003).

In the headspace mode of SDME (HS-SDME), where a microdrop of water or water-miscible organic solvent is used for the extraction of volatile or semi-volatile analytes, any solvent with the ability to extract the analytes can be used, but generally, the high boiling point solvents which have low vapor pressure are preferred. The high surface tension of aqueous solutions also allows the use of relatively large drop volumes. HS-SDME is very useful when liquid chromatography or capillary electrophoresis is involved, as the extraction phase can be compatible with the mobile phase or the electrolyte used in these separation techniques (Psillakis \& Kalogerakis, 2002).

Ionic analytes can be extracted by SDME into organic phase either by addition of a complexing agent to the sample prior to the extraction or by performing the extraction with a microdrop of organic phase containing a complexing agent (Dadfarnia \& Haji Shabani, 2010, Pena-Pereira et al., 2010).

The nature of the analyte and the feasibility of matching between the volume of acceptor phase provided by the SDME technique and the one required for measurement determine which detection system is more suitable. Figure $\mathbf{5 . 1}$ shows the detection techniques used in combination with different mode of SDME methodologies. It can be seen that gas chromatography (GC) is the most common technique combined with SDME, being used in almost $50 \%$ of the studies, followed by high performance liquid chromatography (HPLC) and atomic spectroscopy (AS).

As shown in Figure 5.2, among the organic solvents used as the extractant in SDME techniques, toluene is the most often used solvent especially when the SDME is combined with GC and HPLC, followed by octanol, chloroform, benzene, $n$-hexane, isooctane and hexyl acetate, respectively. Among AS techniques, the electrothermal atomic absorption spectrometry (ETAAS), where a few microliters are sufficient for measurement and the dilution of the enriched extract is avoided, was most commonly used with SDME techniques (ca. $77 \%$ of them). However, it should be noted that when SDME is combined with ETAAS, chlorinated organic solvents are not recommended as 


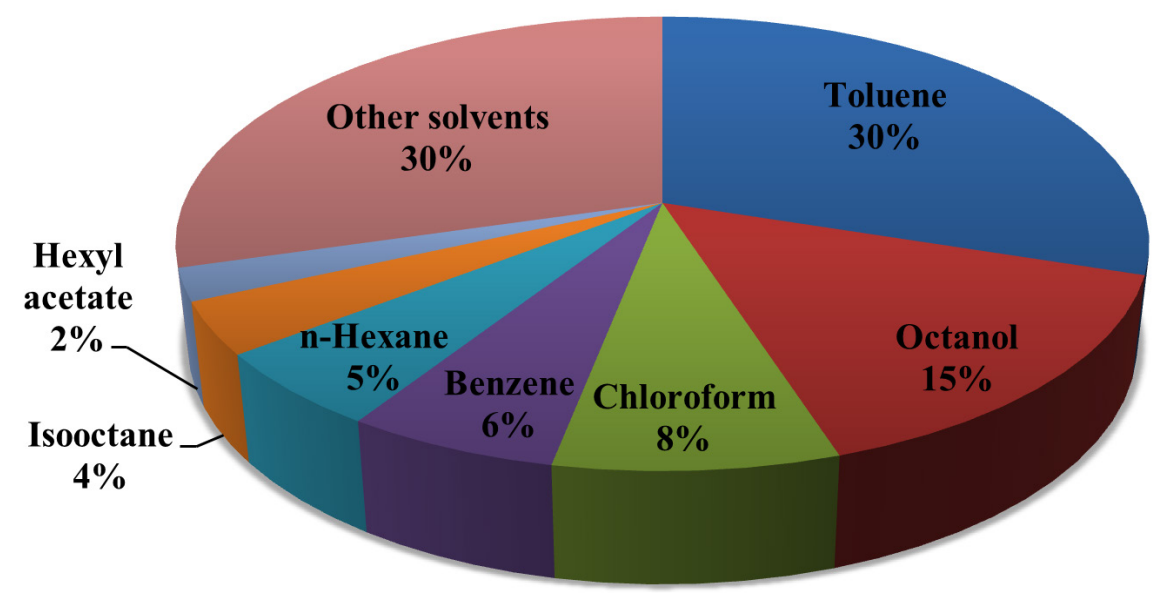

Figure 5.1 Detection techniques used in combination with different modes of SDME methodologies.

extractants due to the possibility of formation of volatile chlorides of analytes in the presence of chlorinated solvent which may result in the significant loss of some analytes. Furthermore, organic solvents may penetrate deeply into the pores of the graphite tube and a high ashing temperature and a long pre-treatment step is required for its removal.

ILs have been used as extracting solvents in both direct-SDME and HS-SDME. Liu et al. reported for the first time the application of ILs in SDME (Liu et al., 2003). Three ILs containing the $\left[\mathrm{PF}_{6}\right]$ anion, 1-alkyl-3-methylimidazolium hexafluorophosphate $\left(\left[\mathrm{C}_{n} \mathrm{MIM}\right]\left[\mathrm{PF}_{6}\right], \mathrm{n}=4,6,8\right)$ were used as the extraction phase coupled to HPLC to determine polycyclic aromatic hydrocarbons (PAHs) in water samples. Compared to organic solvents, larger microdroplets were formed using $\left[\mathrm{C}_{8} \mathrm{MIM}\right]\left[\mathrm{PF}_{6}\right]$, resulting in several orders of magnitude increase in the EFs. Later, ILs with the form $\left[\mathrm{C}_{n} \mathrm{MIM}\right]$ $\left[\mathrm{PF}_{6}\right]$ were used as extractants in both direct-SDME and HS-SDME for the extraction of several compounds, given that they have adequate viscosity and immiscibility in water and nonvolatility (Liu et al., 2004, Peng et al., 2005). The viscosity of ILs plays a critical role in the extraction performance. If the viscosity of ILs is low the microdroplet tends to fall into the sample solution, whereas for more viscous ILs, the microdroplet often traverses up the body of the syringe. Thus, an optimum range of viscosity is needed for a successful extraction.

The main advantages of ILs as extracting solvents for SDME are that they allow the use of larger drop volumes for longer extraction times in comparison with organic solvents, leading to the development of protocols with higher EFs and sensitivity. The negligible volatility and thermal stability of ILs allows their exposure to the headspace of samples heated at high temperature without loss (Peng et al., 2005). In addition to organic compounds, ILs have been also used as extractants in SDME of heavy metal ions from environmental and food samples. In 2009, Manzoori et al. used 


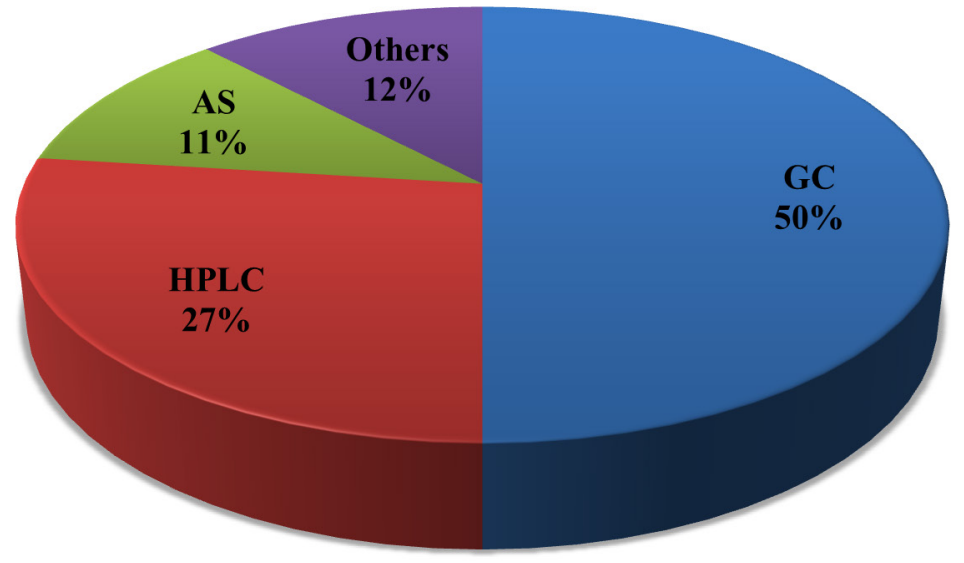

Figure 5.2 Organic solvents used as the extractant in SDME techniques.

IL-SDME for the separation and preconcentration of lead from environmental water samples (Manzoori et al., 2009). Ammonium pyrrolidine dithiocarbamate was used as the complexing agent. The complex was extracted into $7 \mu \mathrm{L}$ of IL and was directly injected into a graphite furnace for quantification.

ILs based on the $\left[\mathrm{PF}_{6}\right]$ anion are the most common ILs used in SDME. However, the limitation of these ILs in SDME is their instability and possibility of dissolution in the sample matrix especially at long extraction times. In 2009, Yao et al. prepared a new class of ILs containing the tris(pentafluoroethyl)trifluorophosphate ([FAP]) anion with imidazolium, phosphonium, and pyrrolidinium cations and used them as extractants in direct immersion SDME for polyaromatic hydrocarbons (PAHs) (Yao, 2009). The extract was analyzed by HPLC. The authors found that the selectivity and sensitivity of the extraction can be monitored by tailoring of ILs using functionalized cations. Thus, with the ILs containing large, hydrophobic cations such as trihexyl(tetradecyl)phosphonium FAP, a high EF can be obtained for extraction of high molecular weight and fused rings PAHs, whereas ILs containing smaller and less hydrophobic cations such as 1-hexyl-3-ethylimidazolium FAP are more suitable for the extraction of smaller and more polar PAHs molecules. The main advantages of these groups of ILs compared to $\left[\mathrm{PF}_{6}\right]-$ and bis(trifluoromethylsulfonyl)imide ([NTf $]$ )-based ILs is their strong hydrophobic nature, high thermal stability and better coordination between cations and anion which permit them to be used for longer sampling times in SDME without the dissolution or loss of the IL drop.

Surfactants dissolved in $\left[\mathrm{C}_{4} \mathrm{MIM}\right][\mathrm{Cl}]$ have also been used as extractants in SDME (Yao et al., 2010). The highest sensitivity was obtained with $\left[\mathrm{C}_{4} \mathrm{MIM}\right][\mathrm{Cl}]-\left[\mathrm{C}_{12} \mathrm{MIM}\right] \mathrm{Br}$ micellar solvent for PAHs with high molecular weights and fused rings, whereas the $\left[\mathrm{C}_{4} \mathrm{MIM}\right][\mathrm{Cl}]-\mathrm{SDS}$ was more sensitive to smaller and more polar molecules. 
For analysis of extracts of IL-based SDME, HPLC is the most often used technique. It should be noted that because of high thermal stability and low pressure, ILs are not compatible with direct injection with GC, the most common technique combined with SDME using organic solvents as the extractant. For direct combination of ILbased SDME with GC, a specially designed interface is required. Aguilera-Herrador et al. (2009) designed a removable interface for GC in which the extracted analytes from the IL microdroplet are transferred to the analytical column but prevent the IL itself from entering the column. The advantages of this design are that the column is not contaminated with IL, the GC system does not require special modification and it is possible to use IL-SDME for extraction and analysis of volatile organic compounds in which analysis by HPLC is not practical.

\subsubsection{Extractant Phases for Directly-suspended Droplet Microextraction}

Although direct-SDME method has the advantages of simplicity, rapidity and low cost, its main limitation is the instability of the organic drop that is held at the tip of a microsyringe needle due to gravity, shear force and flow-field turbulence. The volume of the microdroplet is also limited to $\sim 5 \mu \mathrm{L}$, which results in poor compatibility with some analytical instruments such as HPLC that need larger injection volumes. To overcome these problems, a directly-suspended droplet microextraction (DSDME) method was developed by Yangcheng and co-workers (Yangcheng et al., 2006). In this method, a stirring bar is placed at the bottom of the vial containing the aqueous sample solution and is rotated at a suitable speed to cause a gentle vortex formation. Then, a small volume of the extracting solvent is added to the surface of the sample solution and a single droplet is formed at or near the center of rotation. Compared with SDME, this method has more flexibility in the choice of stirring frequency, stability and the volume of solvent. Sarafraz-Yazdi et al. used a mixture of 90:10 (v/v) toluene/1-octanol as the extraction solvent and combined DSDME with HPLC for the determination of diclofenac (Sarafraz-Yazdi et al., 2008). The same authors also applied DSDME with GC coupled to a flame ionization detector (FID) for the determination of two tricyclic antidepressant drugs, amitriptyline and nortriptyline (Sarafraz-Yazdi et al., 2007), and BTEX compounds (Sarafraz-Yazdi et al., 2009).

The main disadvantage of DSDME is the difficulty of separation of the small volume of the suspended extracting solvent $(<5 \mu \mathrm{L})$ from the aqueous solution. Exact collection of the extracting solvent with a microsyringe is complicated and some water may be transferred into the syringe that can create difficulties for some instruments (e.g., GC-ECD). To overcome this problem, a new extraction method named solidified floating organic drop microextraction (SFODME) was developed by Khalili-Zanjani et al. (2007). In this method, a small volume of an organic solvent with a melting point near room temperature (in the range of $10-30^{\circ} \mathrm{C}$ ) is floated on the surface of the aqueous sample. The solution is stirred for a prescribed time and the sample is trans- 
ferred into an ice bath to solidify the organic droplet. The solidified floating organic drop is then transferred into a small conical vial where it is melted immediately at room temperature. The extracted analyte is then determined by either chromatographic or spectrometric methods.

SFODME has the advantages of simplicity, consumption of a small volume of lowtoxicity organic solvent, good reproducibility, low cost, achievement of high preconcentration factors and suitability for the extraction and analysis from samples with complex matrices. In SFODME, the selection of an appropriate extraction solvent is very important to obtain high recovery and EFs. The ideal extraction solvent must have several characteristics: It must be immiscible with water in order to have good extraction efficiency; it should have low volatility to prevent its loss during the extraction; it should have a lower density than water and high affinity for the analytes; it should have a melting point near room temperature (in the range of $10-30{ }^{\circ} \mathrm{C}$ ); and it must be compatible with the detection system (for example, in combination with chromatographic techniques, its peak must be well-separated from the peaks of analytes). According to these criteria, the organic extraction solvents commonly used in SFODME are presented in Table 5.2 (Ghambarian et al., 2013). 1-undecanol is frequently the solvent of choice to extract metal ions (Dadfarnia et al., 2008; Shirani Bidabadi et al., 2009), while other solvents such as 1-dodecanol and 2-dodecanol have been used to extract organic compounds (Farahani et al., 2008).

Table 5.2 Common used organic solvents in SFODME.

\begin{tabular}{llll}
\hline Extraction solvent & Melting point $\left({ }^{\circ} \mathrm{C}\right)$ & Boiling point $\left({ }^{\circ} \mathrm{C}\right)$ & Density $\left(\mathbf{g ~ c m}^{-3}\right)$ \\
\hline 1-undecanol & $13-15$ & 243 & 0.83 \\
1-dodecanol & $22-24$ & 259 & 0.83 \\
2-dodecanol & $17-18$ & 249 & 0.80 \\
$n$-hexadecane & 18 & 287 & 0.77 \\
1-bromohexadecane & $17-18$ & 190 & 0.99 \\
1-chlorooctadecane & $20-23$ & 157 & 0.85 \\
1,10-dichlorodecane & $14-16$ & 167 & 0.99 \\
\hline
\end{tabular}

The volume of the organic extraction solvent directly influences the extraction efficiency and EF of analytes. By increasing the extraction solvent volume to some extent, the extraction efficiency is increased. However, further increases in the extraction solvent volume will cause an increase in the volume of the final floating organic phase, resulting in dilution of the analytes and a decrease in the EF and sensitivity. Therefore, the volume of extraction solvent should be optimized to reach the maximum sensitivity. In other words, the volume of solvent should be sufficient for subsequent analysis and it should also provide a relatively high EF. For experimental purposes, 5-200 $\mu \mathrm{L}$ of extraction solvent is usually chosen. 


\subsubsection{Extractant Phases for Hollow Fiber Liquid-phase Microextraction}

As was discussed earlier, a successful extraction requires a large distribution coefficient for the analyte between the organic and aqueous phase. In the two-phase HF-LPME sampling mode, the analyte $X$ is extracted from an aqueous sample (donor phase) through the organic phase which is immobilized in the pores of hollow fiber into the acceptor phase inside the lumen of the hollow fiber. The acceptor phase may be the same organic solvent as the one immobilized in the pores of hollow fiber and the analyte $X$ is finally collected in the organic phase. Thus, the extraction process in the two-phase mode can be represented by the following equation (Rasmussen \& Pedersen-Bjergaard, 2004):

$$
X_{\text {sample }} \leftrightarrow X_{\text {organic phase }}
$$

The equilibrium process is dependent on the partition coefficient of the analyte between the organic acceptor solution and the donor solution $\left(K_{a / d}\right)$ which is defined as:

$$
K_{a / d}=C_{\text {aqueous }} / C_{\text {organic }}
$$

where $C_{\text {aqueous }}$ and $C_{\text {organic }}$ are the analyte concentration at equilibrium in the donor solution and the acceptor solution, respectively.

In the three-phase mode of HF-LPME, the acceptor solution may be another aqueous phase in which the analyte $X$ is extracted from an aqueous sample, through the thin film of organic solvent. The equilibria involved are as follows:

$$
X_{\text {sample }} \leftrightarrow X_{\text {organic phase }} \leftrightarrow X_{\text {aqueous acceptor phase }}
$$

The three phases HF-LPME are characterized by $K_{o r g / d}$ and $K_{a / o r g}$ which are the distribution ratio at equilibrium between the organic/donor phases and acceptor solution/organic phase; respectively. The overall distribution ratio $K_{a / d}$ between the acceptor and donor phase can be written as:

$K_{a / d}=K_{o r g / d} \times K_{a / o r g}$

For successful three-phase HF-LPME, the $K_{a / d}$ must be much greater than one. This can be achieved by analyte conversion in the acceptor phase, by exploiting reactions such as complexation or protonation that lead to the formation of analyte species with lower affinity for the organic phase. Thus, in order to achieve a high distribution ratio and develop a sensitive two- or three-phase HF-LPME for efficient analyte separation and preconcentration, the selection of the suitable solvent is a crucial step.

In general, several water-immiscible solvents differing in polarity and water solubility should be tested. It is also possible to use a mixture of organic solvent to obtain the proper solubility parameter $(d)$. Care should also be taken to avoid air bubble formation during immobilization of the solvent in the hollow fiber, as bubbles adhering 
to the surface of the hollow fiber promote solvent evaporation and poor precision. In summary, the final choice of the solvent has to satisfy the following criteria (Rasmussen \& Pedersen-Bjergaard, 2004; Pedersen-Bjergaard \& Rasmussen, 2008):

1. It should be immiscible or have low solubility in water so as to prevent its dissolution into the aqueous phase.

2. The solubility of the analyte in the immobilized solvent should be higher than its solubility in the donor phase to promote analyte migration through the pores of the hollow fiber.

3. The solvent should have low volatility to prevent its evaporation during extraction.

4. The organic solvent should have a polarity matching that of the polypropylene fiber, so that it can be easily immobilized within the pores of the hollow fiber.

5. The solvent should provide an appropriate extraction selectivity to provide high extraction recovery.

6. It should be compatible with the instrument used for the analysis of the final extract.

The most often used solvents are 1-octanol, toluene and dihexyl ether followed by hexane, octane, nonane, dichloromethane, butyl acetate, 2-octanone and diamyl ether. Typically, two-phase HF-LPME is conducted with either toluene or n-octanol as the organic phase, whereas three-phase HF-LPME involves the use of $n$-octanol or dihexyl ether as the support liquid membrane.

ILs have also been used as solvents in HF-LPME. The unique physicochemical properties of ILs such as high stability, high viscosity, tunable polarity, non volatility, good thermal stability, compatibility of its polarity with the polypropylene fiber as well as good extractability for various organic compounds and metal ions make them very useful solvents for HF-LPME. Peng et al. (2007) were the first to support the IL 1-octyl-3-methylimidazolium hexafluorophosphate $\left[\mathrm{C}_{8} \mathrm{MIM}\right]\left[\mathrm{PF}_{6}\right]$ on a hollow fiber for the extraction and determination of chlorophenols in environmental water samples. They reported that, in comparison with traditional organic solvents, the HF-LPME based on ILs was more efficient, easier to operate and had good reproducibility and spiked recovery. In addition, supported ILs are quite stable under mild stirring conditions.

In the three-phase mode of HF-LPME, ILs are immobilized within the pores of the membrane, which acts as a barrier between the donor phase and acceptor phase. Furthermore, ILs of $\left[\mathrm{C}_{4} \mathrm{MIM}\right]\left[\mathrm{PF}_{6}\right]$ or $\left[\mathrm{C}_{6} \mathrm{MIM}\right]\left[\mathrm{PF}_{6}\right]$ have been immobilized in the hollow fiber for the extraction of heavy metal ions such as $\mathrm{Pb}$, Ni and $\mathrm{Cd}$ from water samples (Abulhassani et al., 2010). 


\subsubsection{Extractant Phases for Dispersive Liquid-liquid Microextraction}

DLLME was introduced by Rezaee and co-workers in 2006 (Rezaee et al., 2006). This technique uses a few microliters of a binary mixture of disperser and extraction solvents. A cloudy solution is formed when the mixture is rapidly injected into the aqueous sample containing the analytes of interest with a syringe. The hydrophobic solutes are enriched in the fine droplets of extraction solvent and after centrifugation, determination of the analytes can be performed by conventional instrumental analysis. Thus, the selection of an appropriate extraction solvent is an important parameter for DLLME process. To separate the extraction solvent from the aqueous phase by centrifugation, the extraction solvent should have different densities than the aqueous sample solution (usually higher density). The extraction solvent must have good extraction efficiency for compounds of interest, and low solubility in water. It must be miscible with the dispersive solvent and form a stable cloudy solution. Halogenated hydrocarbons with density higher than water, such as chlorobenzene, bromobenzene, dibromobenzene, chloroform, carbon tetrachloride, and tetrachloroethylene, have been used as DLLME extractants. Other non-chlorinated solvents such as 1-undecanol, 1-dodecanol, 2-dodecanol, $n$-hexadecane, carbon disulphide, 1-octanol, toluene and nitrobenzene have also been used as extraction phases. The application of ILs as extraction solvents in DLLME has become a comtemporary research topic (Han et al., 2012). ILs have been dispersed into the aqueous phase either with a common dispersive solvent such as methanol (Liu et al., 2009) or dispersed and dissolved in aqueous sample by temperature control of the mixture (Zhou et al., 2008).

The dispersive solvent also plays a key role in DLLME. It must be miscible with both the aqueous and organic extraction solvents. In practice, the disperser represents $97-99 \%$ of the total volume of the extraction mixture and helps to form a cloudy solution of fine droplets of extraction solvent in aqueous samples. In comparison with other LPME methods, there is abundant surface contact between fine droplets and the analytes in DLLME, which speeds up the mass transfer process of analytes from the aqueous phase to the organic phase, and the system quickly reaches equilibrium. Due to low toxicity and low cost, acetone, methanol, ethanol and acetonitrile are generally used as the dispersive solvent, while tetrahydrofuran (THF) has also been used. Although THF is more costly and noxious than other dispersive solvents, it may reduce the volume requirement of chlorinated extraction solvents (Guo et al., 2009).

Unlike SDME, a syringe is not applied to hold the extracting solvent drop during the extraction in DLLME, but used only in the collection and injection of the extract. Thus, problems such as drop dislodgment are avoided.

The main advantages of DLLME are simplicity, rapidity, low sample volume, negligible consumption of extracting and dispersing solvents, low cost, high recovery, high EFs and a very short extraction time. The technique can be applied for the determination of various trace organic pollutants and metal ions in different samples. Factors that affect the process include the type and volume of extraction solvent, type 
and volume of dispersive solvent, $\mathrm{pH}$ of the sample solution and the extraction time. In order to achieve good performance, all of these parameters should be optimized.

Jafarvand and Shemirani developed an alternative DLLME method called supramolecular-based dispersive liquid-liquid microextraction (SM-DLLME) (Jafarvand \& Shemirani, 2011). In this method, the analyte is micro-extracted with coacervates composed of reverse micelles made from decanoic acid and dispersed in THF-water mixtures. In comparison with conventional DLLME, SM-DLLME uses decanoic acid, which is a more environmentally friendly solvent.

As stated previously, a suitable disperser solvent has to be miscible with both aqueous and organic phases to ensure the formation of a cloudy solution and to facilitate the extraction process. However, the use of relatively large volume of disperser solvent (mL-level) can be the most significant drawback of DLLME, because it causes a partial dissolution of the analytes in the aqueous phase and decreases the efficiency of extraction. To overcome this drawback, researchers have attempted to perform DLLME without a disperser solvent. In this regard, techniques such as cold induced aggregation microextraction (CIAME), in situ solvent-formation microextraction (ISFME), magnetic stirring-assisted DLLME, ultrasound-assisted DLLME and air-assisted DLLME has been developed. Readers are reffered to chapter 4 for further information on these miniaturized techniques.

CIAME is a modified DLLME procedure (Baghdadi \& Shemirani, 2008). It involves the addition of an IL as the extractant solvent and a non-ionic surfactant as the antisticking agent to an aqueous sample containing the analyte and derivative reagent in a conical-bottom centrifuge tube. IL is dissolved in the aqueous sample by heating the centrifuge tube in a thermostated water bath. After shaking, the centrifuge tube is placed in an ice bath and a cloudy solution like DLLME is formed. Then, the mixture is centrifuged. The fine droplets of ILs are settled in the bottom of the centrifuge tube and are easily separated from the bulk aqueous solution by inverting the tube. The solubility of IL increases with increasing the salt content of the sample. However, according to the common ion effect, the solubility of the IL extractant phase can be decreased by adding another IL with the same ion. Thus, in this method, two different ILs are required to carry out the extraction of analytes in samples with high salt content. Furthermore, the addition of a non-ionic surfactant as anti-sticking agent to the sample prevents the adhesion of ILs to the wall of the tube after centrifugation. Thus, in the presence of a non-ionic surfactant during the phase separation, molecules of the surfactant surround the fine droplets of ILs and decrease their interactions with the wall of the centrifuge tube.

ISFME is another technique derived from DLLME and is based on the in situ formation of a hydrophobic IL in the sample (Baghdadi \& Shemirani, 2009). In ISFME, a hydrophilic IL (e.g., 1-hexyl-3-methylimidazolium tetrafluorobarate) and an ionpairing agent (sodium hexafluorophosphate) are added to the aqueous sample. A cloudy solution results from the formation of the fine droplets of a hydrophobic IL (1-hexyl-3-methylimidazolium hexafluorophosphate). In this mode of DLLME, there is 


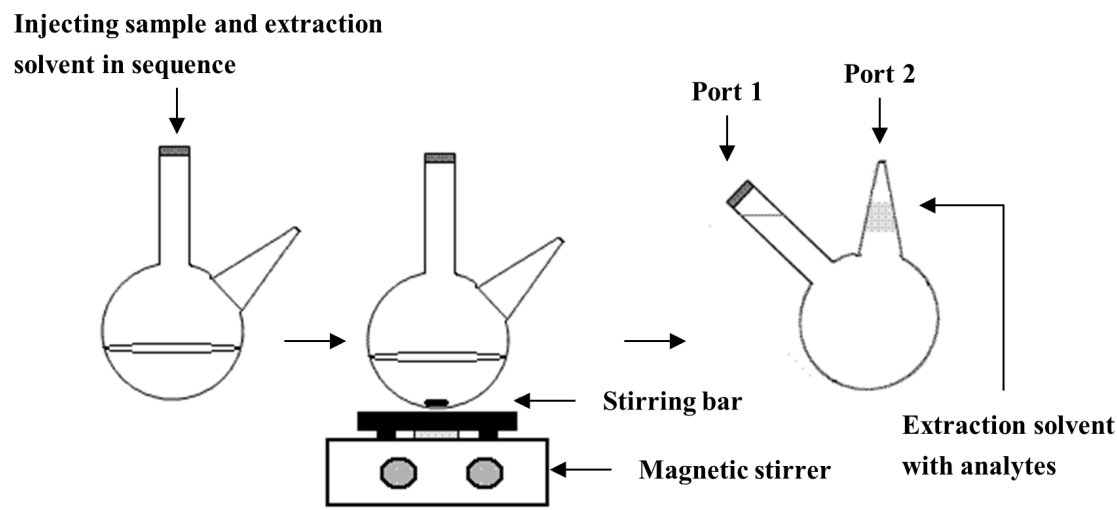

(a)

(b)

(c)

Figure 5.3 Magnetic-assisted DLLME procedure using an extraction solvent that is less dense than water. (A) Extraction device containing aqueous sample and extraction solvent, (B) agitating of the mixture using a magnetic stirrer, (C) elevating the organic phase in the narrow tip of the flask (port 2) by tilting the flask to keep port 2 straight and adding pure water into the flask through port 1.

no interface between the aqueous phase and the extractant phase, and the dispersive solvent is not needed.

Ultrasound radiation in solutions causes acoustic cavitation, which accelerates chemical reactions and mass transfer. Huang et al. first developed an ultrasoundassisted DLLME method to determine NO in cell samples (Huang et al., 2006). In this method, the dispersion of the extractant solvent in the aqueous phase is facilitated by ultrasonic agitation. After the microextraction procedure is complete, a centrifugation step is usually carried out to separate the immiscible phase. This technique is discussed in more detail in chapter 4.

In the magnetic stirring-assisted DLLME method, a binary solvent system of disperser and extraction solvent is avoided. Thus, an extraction solvent having a density lower than water, such as 1-octanol, is added to the aqueous sample solution (Zhang et al., 2011). The extraction phase is dispersed and extraction is accelerated by magnetic agitation of the two phases. After extraction is completed, separation of the organic and aqueous phases is easily obtained by setting aside the extraction system for a period of time. The less dense organic phase, which floats on the surface of the aqueous sample, is then collected via the narrow open tip of the flask upon addition of distilled water into the extraction vessel via other port to raise the volume level (Figure 5.3). The extract is then withdrawn with a microsyringe for the subsequent analysis. The extraction solvent can be easily separated from the aqueous phase without centrifugation, which simplifies the extraction process and make its automation easier.

Air-assisted liquid-liquid microextraction (Figure 5.4) is another mode of DLLME which does not require the use of a disperser solvent. In this mode of extraction the 


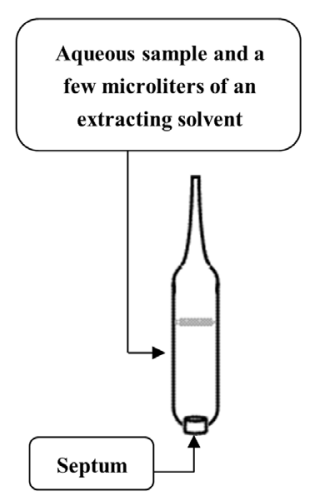

(A)

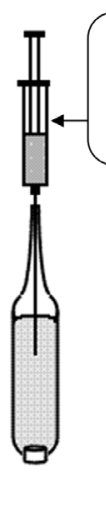

(B)
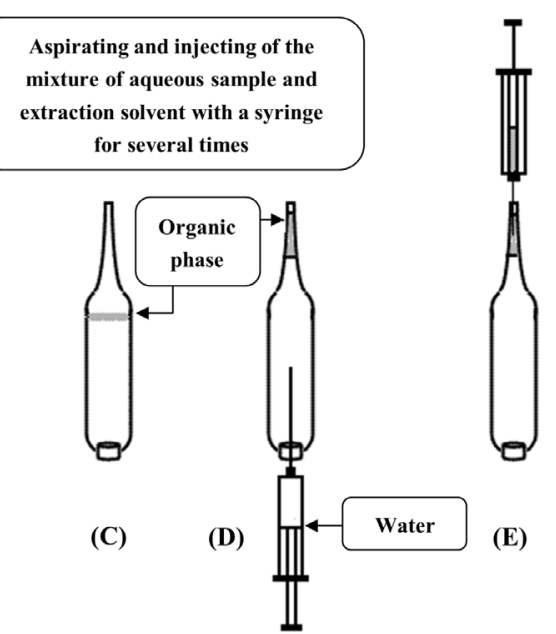

(E)

Figure 5.4 Air assisted liquid-liquid microextraction procedure using an extraction solvent less dense than water. (A) Extraction vessel containing aqueous sample and extraction solvent; (B) aspirating and injecting the mixture of aqueous sample solution and extraction solvent with a syringe several times; (C) collection of organic extraction solvent at the top of aqueous phase after centrifuging; (D) elevating the organic phase by injecting de-ionized water through the septum in the bottom of vessel with a syringe; $(E)$ removal of the collected organic phase in the narrow portion of the tube by the use of a syringe.

extraction solvent must also have a density lower than water (Farajzadeh \& Khoshmaram, 2013). The aqueous sample solution containing the analytes and appropriate amount of a salt are placed into an extracting vessel and then the mixture is repeatedly drawn into a glass syringe and then injected into the extraction vessel. The solution becomes more and more turbid by this aspiration action. After performing the aspiration and injection cycles for a predetermined number of times, the mixture is centrifuged and the fine droplets of extracting solvent float on top of the aqueous phase. Then, by injection of de-ionized water through the septum at the bottom of extraction vessel, the organic drop containing the extract is elevated and so that it can be collected by a syringe.

The main advantage of these methods over the traditional DLLME technique is that by eliminating the disperser solvent, the EFs, extraction recoveries and limit of detections are significantly improved.

\subsection{Conclusions}

In this chapter, a strategy for the selection of solvents for different modes of LPME has been discussed. By proper choice of solvent, LPME may provide high EFs, high extraction recoveries and excellent sample clean-up with short extraction times. Of 
all the desirable properties described, solubility, selectivity, immiscibility, interfacial tension and chemical reactivity are essential for the process to be successfully carried out. The remaining properties that must be given consideration are good engineering work and cost estimation. Furthermore, IL-based LPME methods have become a promising sample preparation technique. The unique and tunable physical and chemical properties of ILs enable the design and synthesis of specific ILs for selective extraction, which expands the application of ILs to LPME technology.

\section{Abbreviations}

$\left[\mathrm{C}_{n} \mathrm{MIM}\right]\left[\mathrm{PF}_{6}\right] \quad \mathrm{n}=4,6,8$,1-alkyl-3-methylimidazolium hexafluorophosphate

AS atomic spectroscopy

BTEX benzene toluene ethylbenzene and xylenes

CIAME cold induced aggregation microextraction

direct-SDME direct single-drop extraction

DLLME dispersive liquid-liquid microextraction

DSDME directly-suspended droplet microextraction

ECD electron capture detector

EF enrichment factor

ETAAS electrothermal atomic absorption spectrometry

FAP tris(pentafluoroethyl)trifluorophosphate

FID flame ionization detection

GC gas chromatography

HF-LPME hollow fiber-liquid phase microextraction

HPLC high performance liquid chromatography

HS-SDME headspace single-drop extraction

ILs Ionic liquids

ISFME in situ solvent-formation microextraction

LPME liquid-phase microextraction

$\mathrm{NTf}_{2} \quad$ bis(trifluoromethylsulfonyl)imide

PAHs polyaromatic hydrocarbons

SDME single-drop extraction

SFODME solidified floating organic drop microextraction

SM-DLLME supramolecular-based dispersive liquid-liquid microextraction

THF tetrahydrofuran. 


\section{References}

Abulhassani, J. Manzoori J. L.,, \& Amjadi M. (2010). Hollow fiber based-liquid phase microextraction using ionic liquid solvent for preconcentration of lead and nickel from environmental and biological samples prior to determination by electrothermal atomic absorption spectrometry. Journal of Hazardous Materials, 176, 481-486.

Aguilera-Herrador, E., Lucena, R., Cárdenas, S., \& Valcárcel, M. (2009). Ionic liquid-based single drop microextraction and room-temperature gas chromatography for on-site ion mobility spectrometric analysis. Journal of Chromatography A, 1216, 5580-5587.

Baghdadi, M., \& Shemirani, F. (2008). Cold-induced aggregation microextraction: A novel sample preparation technique based on ionic liquids. Analytica Chimica Acta, 613, 56-63.

Baghdadi, M., \& Shemirani, F. (2009). In situ solvent formation microextraction based on ionic liquids: A novel sample technique for determination of inorganic species in saline solutions. Analytica Chimica Acta, 634, 186-191.

Barwick, V. J. (1997). Strategies for solvent selection- a literature review. TrAC Trends in Analytical Chemistry, 16, 293-309.

Dadfarnia, S., \& Haji Shabani, A. M. (2010). Recent development in liquid phase microextraction for determination of trace level concentration of metals-A review, Analytica Chimica Acta, 658, 107-119.

Dadfarnia, S., Salmanzadeh, A. M., \& Haji Shabani, A. M. (2008). A novel separation/preconcentration system based on solidification of floating organic drop microextraction for determination of lead by graphite furnace atomic absorption spectrometry. Analytica Chimica Acta, 623, 163-167.

Farahani, H., Yamini, Y., Shariati, S., Khalili Zanjani, M. R., \& Mansour-Baghahi, S. (2008). Development of liquid phase microextraction method based on solidification of floated organochlorine pesticides in water samples. Analytica Chimica Acta, 626, 166-173.

Farajzadeh, M. A., \& Khoshmaram, L. (2013). Air-assisted liquid-liquid microextraction-gas chromatography-flame ionisation detection: A fast and simple method for the assessment of triazole pesticides residues in surface water, cucumber, tomato and grape juices samples. Food Chemistry, 141, 1881-1887.

Ghambarian, M., Yamini, Y., \& Esrafili, A. (2013). Liquid-phase microextraction based on solidified floating drops of organic solvents, Microchimica Acta, 180, 519-535.

Guo, J. H., Li, X. H., Cao, X. L., Li, Y., Wang, X. Z., \& Xu, X. B. (2009). Determination of triclosan, triclocarban and methyl triclosan in aqueous samples by dispersive liquid-liquid microextraction combined with rapid chromatography. Journal of Chromatography A, 1216, 3038-3043.

Han, D., Tang, B., Lee, Y. R., \& Row K. H. (2012). Application of ionic liquid in liquid phase microextraction technology. Journal of Separation Science, 35, 2949-61.

Huang, K. J. Wang, H., Ma, M., Sha, M. L., \& Zhang, H. S. (2006). Ultrasound-assisted liquid phase microextraction and high-performance liquid chromatographic determination of nitric oxide produced in PC12 cells using 1,3,5,7-tetramethyl-2,6-dicarbethoxy-8-(3',4'-diaminophenyl)difluoroboradiaza-s-indacene. Journal of Chromatography A, 1103, 193-201.

Jafarvand, S., \& Shemirani, F. (2011). Supramolecular-based dispersive liquid-liquid microextraction: A novel sample preparation technique for determination of inorganic species. Microchimica Acta, 173, 353-359.

Jeannot, M. A., \& Cantwell F. F. (1996). Solvent microextraction into a single drop. Analytical Chemistry, 68, 2236-2240.

Khalili Zanjani, M. R., Yamini, Y., Shariati, S., \& Jönsson, A. A. (2007). A new liquid-phase microextraction method based on solidification of floating organic drop. Analytica Chimica Acta, 585, 286-293. 
Lide, D. R., ed. (1991-1992) CRC Handbook of Chemistry and Physics (1991-1992) 72 ${ }^{\text {nd }}$ edition, Boca Roaton, Florida, CRC Press.

Liu, J. F., Chi, Y. G., Jiang, G. B., Tai, C., Peng, J. F., \& Hu, J. T. (2003). Use of ionic liquids for liquid-phase microextraction of polycyclic aromatic hydrocarbons, Analytical Chemistry, 75, 5870-5876.

Liu, H., \& Dasgupta P. K. (1996). Analytical chemistry in a drop. Solvent extraction in a microdrop. Analytical Chemistry, 68, 1817-1821.

Liu, J. F., Jiang, G. B., Chi, Y. G., Cai, Y. Q., Zhou, O. X., \& Hu, J. (2004). Ionic liquid-based liquid phase microextraction, a new sample enrichment procedure for liquid chromatography. Journal of Chromatography A, 1026, 143-147.

Liu, Y., Zhao, E., Zhu, W., Gao, H., \& Zhou Z. (2009). Determination of four heterocyclic insecticides by ionic liquid dispersive liquid-liquid microextraction in water samples, Journal of Chromatography A, 1216, 885-891.

Manzoori, J. L., Amjadi, M., \& Abulhassani, J. (2009). Ultra-trace determination of lead in water and food samples by using ionic liquid-based single drop microextraction-electrothermal atomic absorption spectrometry. Analytica Chimica Acta, 644, 48-52.

Pedersen-Bjergaard, A., \& Rasmussen, K. E. (2008). Liquid-phase microextraction with porous hollow fibers, a miniaturized and highly flexible format for liquid-liquid extraction. Journal of Chromatography A, 1184, 132-142.

Pena-Pereira, F., Lavilla, I., \& Bendicho C. (2009) Miniaturized preconcentration methods based on liquid-liquid extraction and their application in inorganic ultratrace analysis and speciation. Spectrochimica Acta Part B: Atomic Spectroscopy, 64, 1-15.

Pena-Pereira, F., Lavilla, I., \& Bendicho C. (2010). Liquid-phase microextraction approaches combined with atomic detection: A critical review. Analytica Chimica Acta 669, 1-16.

Peng, J. F., Liu, J. F., Hu, X. L., \& Jiang, G. B. (2007). Direct determination of chlorophenols in environmental water samples by hollow fiber supported ionic liquid membrane extraction coupled with high-performance liquid chromatography. Journal of Chromatography A, 1139. 165-170.

Peng, J. F., Liu J. F., Jiang, G. B., Tai, C., \& Huang, M. J. (2005). Ionic liquid for high temperature headspace liquid-phase microextraction of chlorinated anilines in environmental water samples. Journal of Chromatography A, 1072, 3-6.

Psillakis, E., \& Kalogerakis, N. (2002). Development in single-drop microextraction. TrAC Trends in Analytical Chemistry, 21, 53-63.

Psillakis, E., \& Kalogerakis, N. (2003). Development in liquid phase microextraction. TrAC Trends in Analytical Chemistry, 22, 565-574.

Rasmussen, K. E., \& Pedersen-Bjergaard, A. (2004). Development in hollow fiber-based, liquid-phase microextraction. TrAC Trends in Analytical Chemistry, 23, 1-10.

Rezaee, M., Assadi, Y., \& Milani Hosseini, M. R. (2006). Determination of organic compounds in water using dispersive liquid-liquid microextraction. Journal of Chromatography A, 1116, 1-9.

Sarafraz-Yazdi, A., Amiri, A. H., \& Es'haghi, Z. (2009). Separation and determination of benzene, toluene, ethylbenzene and 0 -xylene compounds in water using directly suspended droplet microextraction coupled with gas chromatography-flame ionization detector. Talanta, 78, 936-941.

Sarafraz-Yazdi, A., Mofazzeli, F., \& Es'haghi, Z. (2008). Directly suspended droplet three liquid phase microextraction of diclofenac prior to LC. Chromatographia, 67, 49-53.

Sarafraz-Yazdi, A., Raouf-Yazdinejad, S., Es'haghi, Z. (2007). Directly suspended droplet microextraction and analysis of amitriptyline and nortriptyline by GC. Chromatographia, 66, 613-617.

Shirani Bidabadi, M., Dadfarnia, S., \& Haji Shabani, A. M. (2009). Solidified floating organic drop microextraction (SFODME) for simultaneous separation/preconcentration and determination 
of cobalt and nickel by graphite furnace atomic absorption spectrometry (GFAAS). Journal of Hazardous Materials, 166, 291-296.

Swatloski, R. P., Holbrey, J. D., \& Rogers, R. D. (2003). Ionic liquids are not always green: hydrolysis of 1-butyl-3-methylimidazolium hexafluouophosphate, Green Chemistry, 5, 361-363.

Yao, C., Pitner, W. R, \& Anderson, J. L. (2009). Ionic liquids containing the tris(pentafluoroethyl) trifluorophosphate anion: a new class of highly selective and ultra hydrophobic solvents for the extraction of polycyclic aromatic hydrocarbons using single drop microextraction Analytical Chemistry, 81, 5054-5063.

Yao, C., Twu, P., \& Anderson, J. L. (2010). Headspace single drop microextraction using micellar ionic liquid extraction solvents. Chromatographia, 72, 393-402.

Yangcheng, L., Quan, L., Guangsheng, L., \& Youyuan, D. (2006). Directly suspended droplet microextraction. Analytica Chimica Acta, 566, 259-269.

Zhang, P. P., Shi, Z. G., Yu, Q. W., \& Feng, Y. Q. (2011). A new device for magnetic stirring dispersive liquid-liquid microextraction of UV filters in environmental water samples. Talanta, 83, 1711-1715.

Zhou, Q. Bai, H., Xie, G., \& Xiao J. (2008). Temperature-controlled ionic liquid dispersive liquid phase micro-extraction, Journal of Chromatography A, 1177, 43-49. 